\title{
Finsler Manifoldunda Genel Helisler Üzerine Bir Çalışma
}

\author{
Muradiye ÇİMDİKER, Yasin ÜNLÜTÜRK* \\ Kırklareli Üniversitesi, Fen-Edebiyat Fakültesi, Matematik Bölümü, Kırklareli \\ (ORCID:0000-0002-2545-5453) (ORCID:0000-0002-6809-1266)
}

\begin{abstract}
$\ddot{O} \mathbf{z}$
Bu çalışmada, 3-boyutlu Finsler manifoldunda iki özel eğri arasındaki ilişki üzerine çalıştık. 3-boyutlu Finsler manifoldundaki bir regüler eğri ve bir genel helis arasındaki bir denklem kullanılarak, regüler eğri ve genel helis mevcut ise, o zaman regüler eğrinin de bir genel helis olduğunu gösterdik. Daha sonra bu özel eğrilerin her ikisi için de Bertrand çifti, slant helis olma koşulu verildi. Böylece 3-boyutlu Finsler manifoldunda bu eğrilerin bazı karakterizasyonlarını elde ettik.
\end{abstract}

Anahtar kelimeler: Finsler Manifold, Genel Helis, Bertrand Çiftleri.

\section{A Study on the General Helix in Finsler Manifold}

\begin{abstract}
In this study; we worked on the relation between two special curves in 3-dimensional Finsler manifold. By using an equation between the a regular curve and a general helix in 3-dimensional Finsler manifold, we showed that if there exist the regular curve and the general helix, then the regular curve also is a general helix. Then, the condition of being like a slant helix, Bertrand mate for both of them has been given. So, we obtained some characterizations of these special curves in 3-dimensional Finsler manifold.
\end{abstract}

Keywords:Finsler Manifold, General Helix, Bertrand Mates.

\section{Giriş}

"Finsler geometri" isimlendirmesi P. Finsler'in 1918 y1lındaki tezinden gelmektedir. Finsler geometri teorik matematiğin bir alanıdır ve Riemann geometrisinin genelleştirilmelerinden biri olarak kabul edilir. Finsler metriğinin yardımıyla dağın eğimi, anizotropik ortam ve benzeri gibi bazı geometrik modeller elde edilir [1-4].

Eğriler teorisi birçok alanda kullanımını geliştirmeye devam etmesi açısından diferensiyel geometride hala ilginç konulardan biridir ve bugüne kadar bir çok matematikçi tarafından çalışılmaktadır. Eğriler arasında yer alan helis, DNA'nın yapısı, karbon nanotüpleri, nanospringler ve benzeri bir çok uygulamasından dolayı matematikçilerin yanısıra, temel bilimlerin diğer alanlarında çalışna bilim adamlarının da dikkatini çekmiştir [5-7].

Diferensiyel geometride, genel helisin klasik bir sonucu, 1802 y1lında M. A. Lancret tarafindan ifade edilip, 1845 yılında B. de Saint Venant tarafından kanıtlanmıştır [8]. Ayrıca, genel helis ve Öklid 3-uzayındaki ilişkili düzlem eğrileri Sy tarafından çalışılmıştır [9]. Izumiya ve Takeuchi [10] düzlemsel eğriden silindirik helis inşa edilebileceğini göstermişlerdir. Daha sonrasında, Minkowski 3-uzayında genel helis ve ilişkili eğriler çalışılmıştır [11]. 3-boyutlu Finsler manifoldunda da helisler ve Bertrand eğrileri üzerine bazı çalışmalar ve karakterizasyonlar mevcuttur [12,13].

$\mathrm{Bu}$ çalışmada da $[9,14]$ referanslarında verilen iki eğri arasındaki denklem kullanılarak, 3boyutlu Finsler manifoldunda iki eğri arasındaki genel helis, slant helis, Bertrand çifti olma ilişkisi verilmiştir.

*Sorumlu yazar: yasinunluturk@klu.edu.tr

Geliş Tarihi: 17.07.2019, Kabul Tarihi: 17.12.2019 


\section{Materyal ve Metot}

Tanım 2.1. M, m-boyutlu diferensiyellenebilir reel bir manifold ve $T M$ bir tanjant demet olsun. $M$ manifoldu üzerinde $T M$ tanjant demetinin kanonik projeksiyonu $\Pi$ tarafindan gösterilsin. $\Pi\left(M^{\prime}\right)=M$ ve $\theta(M) \cap M^{\prime}=\emptyset$ olacak şekilde $T M$ tanjant demetinin boştan farklı $M^{\prime}$ açı altmanifoldunu gözönüne alalım. Burada $\theta$ sifir kesmedir.

$F: M^{\prime} \rightarrow(0, \infty)$ fonksiyonu bir $C^{2}$ fonksiyon ve $F^{*}=F^{2}$ olsun. $M^{\prime}$ açık altmanifoldu üzerinde $\left\{\left(U^{\prime}, \varphi^{\prime}\right) ; x^{i}, y^{i}\right\}$ herhangi koordinat sistemi için aşağıdaki şartlar sağlanır:

$\left(F_{1}\right)$ Herhangi $(x, y) \in \varphi^{\prime}\left(U^{\prime}\right)$ ve $k>0$ için, $\mathrm{F}$,

$F\left(x^{1}, \ldots, x^{m}, k y^{1}, \ldots, k y^{m}\right)=k F\left(x^{1}, \ldots, x^{m}, y^{1}, \ldots, y^{m}\right)$

herhangi $\left(y^{1}, \ldots, y^{m}\right)$ terimleri için birinci dereceden pozitif homojendir.

$\left(F_{2}\right)(x, y) \in \varphi^{\prime}\left(U^{\prime}\right)$ herhangi bir noktasında

$g_{i j}(x, y)=\frac{1}{2} \frac{\partial^{2} F^{*}}{\partial y^{i} \partial y^{j}}(x, y), \quad i, j \in\{1, \ldots, m\}$

$R^{m}$ üzerinde pozitif tanımlı kuadratik formun bileşenleridir [15].

Böylece, $\left(F_{1}\right)$ ve $\left(F_{2}\right)$ şartlarını sağlayan $F^{m}=\left(M, M^{\prime}, F\right)$ üçlüsüne bir Finsler manifold denir ve $F, F^{m}$ nin bir temel fonksiyonudur.

Tanım 2.2. $F^{m+1}=\left(M, M^{\prime}, F\right)$ bir Finsler manifold ve $F^{m}=\left(\gamma, \gamma^{\prime}, F\right), F^{m+1}$ manifoldunun 1-boyutlu Finsler altmanifoldu olsun. Burada $\gamma, M$ de

$$
x^{i}=x^{i}(s), \quad i \in\{1, \ldots, m+n\}, \quad s \in(a, b)
$$

olarak verilen $s$ yay parametreli bir $C^{2}$ eğrisidir. $\gamma^{\prime}$ üzerindeki koordinatları $(s, v)$ tarafindan gösterilir. O zaman, $y^{i}(s, v)=v \frac{d x^{i}}{d s}, \quad i \in\{0, \ldots, m\}$ olarak elde edilir.

Dahası, $\gamma$ eğrisi üzerinde $\left\{\frac{\partial}{\partial s}, \frac{\partial}{\partial v}\right\}$ doğal bir çatı alanıdır. Burada $\frac{\partial}{\partial v}$ bir birim Finsler vektör alanıdır [15].

Tanım 2.3. $F^{3}=\left(M, M^{\prime}, F\right)$ bir 3-boyutlu Finsler manifold ve $\gamma$,

$x^{i}=x^{i}(s),\left(x^{\prime 1}(s), x^{\prime 2}(s), x^{\prime 3}(s)\right) \neq(0,0,0)$ olarak verilen, $s$ yay parametreli, $M$ de $C^{2}$ eğrisi olsun.

O zaman,

$$
\begin{aligned}
\nabla_{\frac{\partial}{\partial s}}^{*} \frac{\partial}{\partial v} & =\kappa N, \\
\nabla_{\frac{\partial}{\partial s}}^{*} N & =-\kappa \frac{\partial}{\partial v}+\tau B, \\
\nabla_{\frac{\partial}{\partial s}}^{*} B & =-\tau N
\end{aligned}
$$

elde edilir. Burada, $N$ ve $B$, sirasıyla, birim normal Finsler vektör ve binormal Finsler vektör alanıdır. 3boyutlu Finsler manifoldunda, $\gamma$ eğrisi için $\left\{\frac{\partial}{\partial v}, N, B\right\}$ Frenet çatıyı gösterir. Riemann durumda, $\kappa$ ve $\tau$, $\gamma$ eğrisinin, sırasıyla, eğrilik ve torsiyonudur [15].

Tanım 2.4. 3-boyutlu Finsler manifoldunda, $\gamma$ bir eğri ve $\left\{\frac{\partial}{\partial v}, N, B\right\}, \gamma$ eğrisi boyunca Frenet çatısı olsun. Eğer, $\gamma$ eğrisi boyunca $\kappa$ eğriliği ve $\tau$ torsiyonu birer pozitif sabitler ise, o zaman $\gamma$ eğrisi Frenet çatı elemanlarının yardımıyla bir silindirik helis olarak adlandırılır [12]. 
Tanım 2.5. 3-boyutlu Finsler manifoldunda, $\gamma$ bir eğri ve $\left\{\frac{\partial}{\partial v}, N, B\right\}, \gamma$ eğrisi boyunca Frenet çatı olsun. O zaman $\frac{\kappa}{\tau}=s b t$. olacak şekilde, $\gamma$ eğrisi, 3-boyutlu Finsler manifoldunda Frenet çatı elemanları yardımıyla bir genel helis olarak adlandırılır. Burada, $\gamma$ eğrisi boyunca $\kappa$ bir eğrilik ve $\tau$ bir torsiyondur [12].

Tanım 2.6. $F^{m}$ Finsler manifoldu üzerinde, $\gamma$ ve $\gamma^{*}$ eğrileri regüler eğri, $\left\{n_{0}^{i}(s), n_{1}^{i}(s), \ldots, n_{r}^{i}(s)\right\}$ ve $\left\{n_{0}^{i^{*}}(s), n_{1}^{i^{*}}(s), \ldots, n_{r}^{i^{*}}(s)\right\}$, sırasıyla, bu eğriler üzerinde Frenet r-çatılar olsun. $n_{0}^{i}(s)$ ve $n_{0}^{i^{*}}(s)$, lineer bağımsız olduğunda, $\gamma$ ve $\gamma^{*}$ eğrileri, Bertrand eğriler olarak adlandılır. $\gamma^{*}$ eğrisi $\gamma$ için bir Bertrand eştir ve $\left(\gamma, \gamma^{*}\right)$ bir Bertrand çift olarak adlandılır [13].

\section{Bulgular ve Tartışma}

3-boyutlu Finsler manifoldunda, $\alpha^{*}(s)$ bir regüler eğri ve $\alpha(s)$,

$u(s)=\cos \theta \frac{\partial}{\partial v}+\sin \theta B$

eksenli birim hızlı bir genel helis olsun. Burada $s$ yay parametresi ve $\theta, u$ ve $\frac{\partial}{\partial v}$ arasında bir açı, $\left\{\frac{\partial^{*}}{\partial v}, N^{*}, B^{*}\right\}$ ve $\left\{\frac{\partial}{\partial v}, N, B\right\}$, sırasıyla, bu eğrilerin Frenet çatılarıdır.

$\alpha^{*}$ ve $\alpha$ eğrilerinin eğrilik fonksiyonları, sırasıyla, $\kappa^{*}$ ve $\kappa$, torsiyon fonksiyonları $\tau^{*}$ ve $\tau$ dur.

$\alpha^{*}$ ve $\alpha$ eğrileri arasındaki denklem

$$
\alpha^{*}=\alpha\left(s_{0}\right)+\sin \theta \alpha(s)+\left(s-s_{0}\right) \cos \theta u(s)
$$

tarafindan gösterilir. Burada $\alpha\left(s_{0}\right)$ ve $s_{0}$, sirasıyla, keyfi sabit vektör ve noktadır.

(2) denkleminin diferensiyeli alınırsa, o zaman $\alpha^{*}$ eğrisinin teğet vektörü aşağıdaki gibi elde edilir:

$\frac{\partial}{\partial v}^{*}(s)=\frac{\sin \theta+\cos ^{2} \theta}{\sqrt{1+\cos \theta+\sin 2 \theta}} \frac{\partial}{\partial v}(s)+\frac{\cos \theta \sin \theta}{\sqrt{1+\cos \theta+\sin 2 \theta}} B(s)$.

$B(s)=\frac{\alpha \prime(s) \times \alpha \prime \prime(s)}{\|\alpha \prime(s) \times \alpha \prime \prime(s)\|}$ olduğundan,

$B^{*}(s)=-\frac{\lambda}{\sqrt{\lambda^{2}+\mu^{2}}} \frac{\partial}{\partial v}(s)+\frac{\mu}{\sqrt{\lambda^{2}+\mu^{2}}} B(s)$

elde edilir. Burada

$\mu=\kappa\left(\sin \theta+\cos ^{2} \theta\right)^{2}+\tau\left(\cos \theta \sin ^{2} \theta+\cos ^{3} \theta \sin \theta\right)$

ve

$\lambda=\kappa\left(\cos \theta \sin ^{2} \theta+\cos ^{3} \theta \sin \theta\right)+\tau(\cos \theta \sin \theta)^{2}$

dir.

(3) ve (4) denklemlerinden, $\alpha^{*}$ eğrisinin asli normal vektörü

$N^{*}=-\left(\frac{\lambda}{\sqrt{\lambda^{2}+\mu^{2}}} \frac{\cos \theta \sin \theta}{\sqrt{1+\cos \theta+\sin 2 \theta}}+\frac{\mu}{\sqrt{\lambda^{2}+\mu^{2}}} \frac{\sin \theta+\cos ^{2} \theta}{\sqrt{1+\cos \theta+\sin 2 \theta}}\right) N$

olarak bulunur. 
$c=-\left(\frac{\lambda}{\sqrt{\lambda^{2}+\mu^{2}}} \frac{\cos \theta \sin \theta}{\sqrt{1+\cos \theta+\sin 2 \theta}}+\frac{\mu}{\sqrt{\lambda^{2}+\mu^{2}}} \frac{\sin \theta+\cos ^{2} \theta}{\sqrt{1+\cos \theta+\sin 2 \theta}}\right)$

olduğundan, (7) ve (8) denklemlerine göre, $N^{*}(s)=|c| N(s)$ olarak yazılır. Böylece aşağıdaki teoremler verilebilir:

Teorem 3.1. 3-boyutlu Finsler manifoldunda, $\alpha^{*}(s)$ bir regüler eğri ve $\alpha(s)$ birim hizlı genel bir helis olsun. Ĕger $\alpha(s)$ eğrisi genel bir helis ise, bu takdirde $\alpha^{*}(s)$ eğrisi de bir genel helistir.

İspat. (1) denklemine göre, $\left\langle u, \frac{\partial}{\partial v}\right\rangle=\cos \theta$ ve $\langle u, B\rangle=\sin \theta$ eşitliklerine ulaş1lır. Biliyoruz ki, genel helisin teğeti genel helisin ekseniyle sabit bir açı yapar. Ayrıca $\alpha^{*}(s)$ eğrisinin teğet vektörü $\frac{\partial^{*}}{\partial v}, \alpha(s)$ eğrisinin $\frac{\partial}{\partial v}$ teğet vektörüne ve $B$ binormal Finsler vektörüne bağlıdır. Dolayısıyla

$\left\langle u, \frac{\partial}{\partial v}^{*}\right\rangle=\frac{\cos \theta \sin \theta+\cos ^{3} \theta}{\sqrt{1+\cos \theta+\sin 2 \theta}}+\frac{\cos \theta \sin ^{2} \theta}{\sqrt{1+\cos \theta+\sin 2 \theta}}$

biçiminde bulunur.

$\alpha^{*}(s)$ eğrisinin teğet vektörünün, $\frac{\partial^{*}}{\partial v}$ sabit bir vektörle sabit açı yaptığı görülür. Böylece 3-boyutlu Finsler manifoldunda $\alpha^{*}(s)$ eğrisi bir genel helistir.

Teorem 3.2. 3-boyutlu Finsler manifoldunda, $\alpha^{*}(s)$ bir regüler eğri ve $\alpha(s)$ birim hizlı genel bir helis olsun. Ĕger $\alpha(s)$ ve $\alpha^{*}(s)$ eğrilerinin asli normalleri arasında yer alan denklemdeki $c$ sayısı sabit ise, o zaman $\alpha(s)$ eğrisi bir slant helistir ancak ve ancak $\alpha^{*}(s)$ eğrisi bir slant helistir.

İspat. (7) denkleminden, $N^{*}(s)=|c| N(s)$ eşitliğine sahibiz. Bu durumda, eğer $c$ sabit ise, o zaman $n^{*}$ ve $n$ asli normal vektörleri lineer bağımsızdır.

3-boyutlu Finsler manifoldunda, $\alpha(s)$ eğrisi bir slant helis olsun. $\alpha(s)$ eğrisinin asli normal vektörü olan $N$ vektörü sabit bir doğruyla sabit açı yapar. $N^{*}(s)=|c| N(s)$ olduğundan, $\alpha^{*}(s)$ eğrisinin asli normal vektörü olan $N^{*}$ vektörü de sabit bir doğruyla sabit bir açı yapar. Böylece ispat tamamlanır.

Teorem 3.2 ye göre, aşağıdaki gibi bir sonuca ulaşılır:

Sonuç 3.3. $\kappa$ ve $\tau$ fonksiyonları sabitse, 3-boyutlu Finsler manifoldunda, $\alpha(s)$ eğrisi bir silindirik helistir. Eğer $\alpha(s)$ eğrisi bir silindirik helis ise, o zaman $\alpha(s)$ eğrisi bir slant helistir ancak ve ancak $\alpha^{*}(s)$ eğrisi bir slant helistir.

Teorem 3.4. 3-boyutlu Finsler manifoldunda, $\alpha^{*}(s)$ bir regüler eğri ve $\alpha(s)$ birim hızlı genel bir helis olsun. Eğer $\alpha(s)$ ve $\alpha^{*}(s)$ eğrilerinin asli normalleri arasında yeralan denklemdeki $c$ sayısı sabit ise, o zaman, $\alpha(s)$ ve $\alpha^{*}(s)$ birer Bertrand eştir.

İspat. $N^{*}(s)=|c| N(s)$ olduğundan, o zaman $N^{*}$ ve $N$ asli normaller lineer bağımsızdır. Burada $c$ bir sabit sayıdır. Dolayısıyla, $\alpha(s)$ ve $\alpha^{*}(s)$ eğrileri Bertrand eştir.

Teorem 3.4 e göre, aşağıdaki gibi bir sonuca ulaşılır:

Sonuç 3.5. $c$ sayısı sabit olduğundan, o zaman $\alpha(s)$ eğrisi 3-boyutlu Finsler manifoldunda bir silindirik helistir. Eğer $\alpha(s)$ eğrisi bir silindirik helis ise, o zaman $\alpha(s)$ ve $\alpha^{*}(s)$ eğrileri birer Bertrand eştir.

Örnek 3.6. 3-boyutlu Finsler manifoldunda $F(x, y)=\sqrt{x_{1}^{2}+x_{2}^{2}+x_{3}^{2}}+b x_{1}, 0<b<1$ Randers normu olmak üzere orjin merkezli 1 yarıçaplı silindir denklemi

$$
F S=\left(\frac{\cos u-b}{1-b^{2}}, \frac{\sin u}{\sqrt{1-b^{2}}}, v\right)
$$


üzerindeki silindirik helis denklemi

$$
\alpha(s)=\left(\frac{\cos s-b}{1-b^{2}}, \frac{\operatorname{sins}}{\sqrt{1-b^{2}}}, s\right)
$$

olarak verilsin $[16,17]$. Bu helisin ilişkili düzlem eğrisini bulunuz.

Çözüm. Öncelikle, 3-boyutlu Finsler manifoldunda silindirik helis için $\kappa$ ve $\tau$ değerlerini hesaplayalım.

$\alpha^{\prime}(s)=\left(-\frac{\operatorname{sins}}{1-b^{2}}, \frac{\operatorname{coss}}{\sqrt{1-b^{2}}}, 1\right)$

olarak bulunur.

$\alpha(s)$ eğrisi boyunca birim teğet vektörü

$\frac{\partial}{\partial v}=\frac{1}{P}\left(-\sin s, \sqrt{1-b^{2}} \operatorname{coss}, 1-b^{2}\right)$

şeklinde elde edilir. Burada $P=\sqrt{\sin ^{2} s+\cos ^{2} s\left(1-b^{2}\right)+\left(1-b^{2}\right)^{2}}-b \operatorname{sins}$ dir.

$\alpha(s)$ silindik helisinin asli binormal vektörü aşağıdaki gibi elde edilir:

$B=\frac{1}{R}\left(\left(1-b^{2}\right) \operatorname{sins},-\sqrt{1-b^{2}} \operatorname{coss}, 1\right)$

Burada $\quad R=\sqrt{\sin ^{2} s\left(1-b^{2}\right)^{2}+\cos ^{2} s\left(1-b^{2}\right)+1}+\left(b-b^{3}\right) \operatorname{sins} \quad$ dir. Buradan yapilan hesaplamalar sonucunda $\kappa=\frac{R\left(1-b^{2}\right)^{\frac{3}{2}}}{P^{3}}$ ve $\tau=\frac{\left(1-b^{2}\right)^{\frac{3}{2}}}{R^{2}}$ olarak elde edilir. $\frac{\kappa}{\tau}=\left(\frac{R}{P}\right)^{3}=1$ bulunur. $\frac{\kappa}{\tau}=\tan \theta$ olduğundan, $\theta=\frac{\pi}{4}$ sonucuna ulaşılır.

$\alpha(s)$ silindirik helisinin ekseni $u(s)=\cos \theta \frac{\partial}{\partial v}+\sin \theta B$ şeklinde ifade edilir. Dolayısıyla, $\alpha(s)$ silindirik helisinin ekseni

$$
u(s)=\frac{\sqrt{2}}{2}\left[\frac{1}{P}\left(-\sin s, \sqrt{1-b^{2}} \cos s, 1-b^{2}\right)+\frac{1}{R}\left(\left(1-b^{2}\right) \sin s,-\sqrt{1-b^{2}} \operatorname{coss}, 1\right)\right]
$$

olarak bulunur. O zaman

$$
\alpha^{*}(s)=\frac{\sqrt{2}}{2}\left[\begin{array}{c}
\left(\frac{\cos s-b}{1-b^{2}}, \frac{\sin s}{\sqrt{1-b^{2}}}, s\right) \\
+s\left(\frac{1}{P}\left(-\sin s, \sqrt{1-b^{2}} \cos s, 1-b^{2}\right)+\frac{1}{R}\left(\left(1-b^{2}\right) \sin s,-\sqrt{1-b^{2}} \cos s, 1\right)\right)
\end{array}\right]
$$

şeklinde elde edilen bir eğridir. Burada $\alpha\left(s_{0}\right)=0$ keyfi sabit bir vektör ve $s_{0}=0$ bir noktadır.

\section{Sonuçlar}

Bu çalışmada, öncelikle, 3-boyutlu Finsler manifoldunda genel helis, silindirik helis ve Bertrand eşler gibi bazı tanımları verildi. Sonrasında, iki eğri arasındaki geçiş denklemi kullanılarak 3-boyutlu Finsler manifoldunda genel helis olma ilişkisi elde edildi ve bu eğrilerle ilgili bazı sonuçlara ulaşıldı.

\section{Yazarların Katkısı}

Çalışmaya tüm yazarlar eşit oranda katkı sunmuştur. 


\section{Çıkar Çatışması Beyanı}

Yazarlar arasında herhangi bir çıkar çatışması bulunmamaktadır.

\section{Araştırma ve Yayın Etiği Beyanı}

Yapılan çalışmada araştırma ve yayın etiğine uyulmuştur.

\section{Kaynaklar}

[1] Matsumoto M. 1989. A Slope of a Mountain is a Finsler Surface with respect to a Time Measure. Kyoto Journal of Mathematics, 29 (1): 17-25.

[2] Antonelli P.L., Ingarden R.S., Matsumoto M. 1993. The Theory of Sprays and Finsler Spaces with Applications in Physics and Biology. Kluwer Academic Publishers, Dordrecht, Netherlands, 305p.

[3] Bao D., Chern S.S., Shen Z. 2000. Introduction to Riemann-Finsler Geometry, Series: Graduate Texts in Mathematics 200. Springer-Verlag New York, 434p.

[4] Y1lmaz M.Y., Bektas M., Kücükarslan Z. 2012. Siacci's Theorem for Curves in Finsler Manifold $\mathrm{F}^{3}$. Turkish Journal of Science and Technology, 7 (2): 181-185.

[5] Yin Y., Zhang T., Yang F., Qiu X. 2008. Geometric Conditions for Fractal Supercarbon Nanotubes with Strict Self-Similarities. Chaos Solitons and Fractals, 37: 1257-1266.

[6] Jain A., Wang G., Vasquez K.M. 2008. DNA Triple Helices: Biological Consequences and Theropeutic Potential. Biochimie, 90 (8): 1117-1130.

[7] Camcı Ç., İlarslan K., Kula L., Hacısalihoğlu H.H. 2009. Harmonic Curvatures and Generalized Helices in $\mathrm{E}^{\mathrm{n}}$. Chaos Solitons and Fractals, 4: 2590-2596.

[8] Struik D.J. 1988. Lectures on Classical Differential Geometry. Dover, New York, 256p.

[9] Sy S. 2001. General Helices and Other Topics in Differential Geometry of Curves. Michigan Technological University, Master Thesis of Science in Mathematics (Printed), 69p.

[10] Izumiya S., Takeuchi N. 2002. Generic Properties of Helices and Bertrand Curves. Journal of Geometry, 74: 97-109.

[11] Güven İ.A., Kaya S., Yaylı Y. 2010. General Helix and Associated Curve in Minkowski 3-Space. Far East Journal of Mathematical Sciences, 47 (2): 225-233.

[12] Yılmaz M.Y., Bektaş M. 2009. Helices of the 3-Dimensional Finsler Manifolds. Journal of Advanced Mathematical Studies, 2 (1): 107-212.

[13] Yılmaz M.Y., Bektaş M. 2011. Bertrand Curves on Finsler Break Manifolds. International Journal of Physical and Mathematical Sciences, 5-10.

[14] Güven İ.A.,Yaylı Y. 2013. The Helix Relation Between Two Curves. Turkish Journal of Analysis and Number Theory, 1 (1): 23-25.

[15] Bejancu A., Farran H.R. 2000. Geometry of Pseudo-Finsler Submanifold. Kluwer Academic Publishers, Dordrecht, Netherlands, 207p.

[16] Ateş F., Özdemir Z., Ekmekçi F.N. 2018. Special Curves in Finsler Space. Proceedings. Inst. Math. Mechanics, 44 (2): 198-208.

[17] Özdemir Z., Ateş F., Ekmekçi F.N. 2019. Spherical Curves in Finsler 3-Space. Conference Proceeding of Science and Technology, 2 (2): 158-163. 